\title{
L'éducation physique comme analyseur de l'histoire de la mixité dans les écoles (1882-2008)
}

Jacques Gleyse

\section{(2) OpenEdition}

1 Journals

Édition électronique

URL : http://journals.openedition.org/trema/1153

DOI : 10.4000/trema.1153

ISSN : 2107-0997

Éditeur

Faculté d'Éducation de l'université de Montpellier

Édition imprimée

Date de publication : 1 juin 2010

Pagination : 69-92

ISSN : 1167-315X

\section{Référence électronique}

Jacques Gleyse, «L'éducation physique comme analyseur de l'histoire de la mixité dans les écoles

(1882-2008) », Tréma [En ligne], 32 | 2010, mis en ligne le 01 juin 2012, consulté le 01 mai 2019. URL : http://journals.openedition.org/trema/1153; DOI : 10.4000/trema.1153

Ce document a été généré automatiquement le 1 mai 2019.

Trema 
L'éducation physique comme analyseur de l'histoire de la mixité dans les écoles (1882-2008)

\author{
Jacques Gleyse
}

« Ni la femme ni le génie ne travaillent. La femme a été jusqu'à présent le plus haut luxe de l'humanité. À tous les instants où nous produisons

le meilleur de nous-mêmes, nous ne travaillons pas. Le travail n'est qu'un moyen d'atteindre à ces instants » NIETZSCHE, F. Ainsi parlait Zarathoustra, Paris, Gallimard, réed. 1963. Aphorisme 92, p. 403. 
Un récent rapport du Sénat (2004) pose le problème de la mixité à l'Ecole et, pour ce faire, réalise un historique de la « mixité », pour le moins surprenant pour le non spécialiste de l'Histoire de l'Éducation. Ce rapport distingue très bien les notions de co-éducation (véritable prise en compte des différences sexuelles dans l'éducation, ou a minima éducation commune des filles et des garçons), de ceux de co-instruction ou de gémination (mise en présence des sexes liée à des contraintes spatiales, numériques ou économiques). Il note aussi que la notion de mixité est assez spécifique à la France et que dans les autres pays européens c'est généralement le terme de co-éducation qui est privilégié. La mixité serait-elle plus, moins ou autre que la coéducation?

Plusieurs colloques (sciences de l'éducation, sociologie, psychologie, histoire, linguistique ...) ont tenté de démêler le fil d'Ariane de la mixité. L'un d'entre eux, qui s'est tenu à Montpellier, intitulé : « Mixités » (GAUTHIEZ-RIEUCAU, D., 2005) mettait en évidence le spectre large du vocable en ne le limitant pas aux sexes et aux stéréotypes de genre, s'intéressant à la question de la mixité sociale. Lors de ce colloque, a été posée la question de la mixité comme métissage (comme Métis au sens grec du terme). Il a été alors rappelé que la mixité sexuelle pourrait dépasser la co-éducation en réalisant un métissage surplombant des stéréotypes masculins et féminins de genre. Cette mixité métissage contribuerait à aider à analyser les stéréotypes de genre et à les mettre à distance.

3 Elle correspondrait bien à l'étymologie grecque de métissage et au mythe de l'Océanide qui est à l'origine du terme. Métis (littéralement : « le conseil, la ruse ») est celle qui sait plus de choses que tout dieu ou mortel, elle est la personnification de la sagesse et de l'intelligence rusée. Elle est toujours représentée sous le siège de Zeus. Le métissage de genre ou mixité sexuelle contribuerait à un dépassement du concept de stéréotype de genre.

4 Une telle conception de la mixité, fondée sur l'étymologie de Métis, ouvre un certain nombre de portes pour comprendre la différence qu'il peut y avoir entre éduquer côte à côte et éduquer ensemble les deux sexes.

Soulignons aussi immédiatement que l'étymologie du mot mixité, renvoie au latin mixtus (supin : mixtum, issu de misceo), qui signifie mêler, mélanger. Or qu'y aurait-il à mêler dans la mixité, certainement pas, au sens physique et biologique, les sexes puisque l'école (laquelle ?) depuis PLATON (voir le noli tangere : SOCRATE, ALCIBIADE et AGATHON, dans Le Banquet ou plus usuel : le Christ et Marie-Madeleine (Noli me tangere), est formalisée sur le déni de la sexualité et du sexe (LAPASSADE \& SCHERRER, 1974, FILLOUX, 1976, PUJADE-RENAUD, 1983). Très probablement s'agit-il davantage du genre, c'est-à-dire de

Tréma, 32 | 2011 
l'aspect social et culturel de la partition et de la distinction masculin, féminin (et non mâle, femelle). Dans ce cas, le mixage dans l'école peut effectivement se percevoir comme Métis.

C'est dans ce cadre que le monde de l'éducation physique scolaire, fondé sur des pratiques corporelles, est un excellent analyseur pour étudier l'histoire de la mixité dans l'école.

7 Ce qui suit va s'attacher à mettre en liaison les transformations qui se sont produites dans le domaine de l'éducation physique scolaire et celles des écoles en général. Cette étude comparative permettra à la fois de clarifier les notions de co-instruction, mais aussi celles de coéducation, de mixité ainsi que celle de « stéréotype de genre » dans l'école. En effet, si l'on admet avec Michel BERNARD que «le corps est un fait social total» (BERNARD, 1974, p. 141) on comprend que la place de l'éducation des corps féminins et masculins dans l'école puisse être un excellent analyseur des rapports des écoles aux deux sexes.

\section{Ils voient des hommes partout !}

Même si Julie DAUBIE, avec l'appui de l'Impératrice, obtient à Lyon en 1862, son baccalauréat ès lettres dont on lui a refusé la passation à Paris l'année précédente et devient par là même la première femme à accéder à ce grade, il est peu probable qu'elle ait dû valider une note en gymnastique pour obtenir ce diplôme. Le fait également que Madeline BRES, également avec le soutien de l'Impératrice, ait pu entreprendre des études de médecine, la même année, ne doit pas laisser envisager que la mixité dans l'enseignement secondaire a commencé réellement (LELIEVRE, F. \& C., 1991).

9 Il y a bien, à ce moment, dans l'enseignement secondaire, comme dans la pratique des gymnastiques et des tout premiers balbutiements du sport, rejet des filles. Officiellement, les lois FALLOUX (15 mars 1850), même si elles ont permis la mise en œuvre d'une éducation des filles, renforcent la logique selon laquelle celles-ci sont avant tout élevées "sur les genoux de l'église", selon l'expression de Mgr DUPANLOUP. L'Etat n'a pratiquement aucune prise sur l'éducation des femmes, comme il n'a aucune prise sur l'éducation physique de celle-ci. Leur alphabétisation est très inférieure à celle des hommes dans l'ensemble des classes sociales, tout comme leurs pratiques physiques (si l'on exclut le travail productif pour les classes laborieuses, ce qui n'est pas rien), leur pratique de la gymnastique et du sport est quasiment inexistante.

10 La création des Écoles Normales de filles dès 1842, puis la loi Paul BERT (le 9 Août 1879) et les lois FERRY notamment celle du 28 Mars 1882, rendent obligatoires l'enseignement primaire pour les enfants des deux sexes, tout comme ils rendent l'éducation physique obligatoire pour les enfants des deux sexes :

11 «L'instruction primaire est obligatoire pour les enfants des deux sexes âgés de six ans révolus; elle peut être donnée soit dans les établissements d'instruction primaire ou secondaire, soit dans les écoles publiques ou libres, soit dans les familles par le père de famille lui-même ou toute personne qu'il aura choisie » (Article 4).

Au même moment, dans le domaine de l'éducation physique, de profonds changements sont en cours, mais ils vont tout d'abord toucher le système primaire et primaire supérieur. En effet, comme le constate le Baron Pierre DE COUBERTIN, en 1908, l'enseignement secondaire résiste vivement aux pratiques d'exercices physiques et, a fortiori, aux pratiques d'exercices physiques pour les filles. Mais, en fait, c'est toute la société française qui résiste à cela. Plusieurs raisons peuvent l'expliquer, d'une part, le 
fait que l'exercice physique de loisir (à part les jeux traditionnels lors de fêtes paysannes) n'est pas présent pour les couches populaires (qui vont être touchées par l'école obligatoire, laïque et gratuite après l'avoir été par les Écoles des Frères de Jean-Baptiste de LASSALLE), mais aussi le fait que ce sont les hommes qui vont être les premiers pratiquants sportifs et les premiers héros sportifs de l'aristocratie et de la bourgeoisie, mais aussi du peuple avec la création du Tour de France (1903). On voit tout de suite que ce ne sont que des hommes ou presque qui pratiquent le sport dans la société et que tous les héros sportifs, à de très rares exceptions près, sont des hommes. Officiellement l'École, n'accordant qu'une toute petite place aux femmes, s'organise sur la même logique « archéologique » (Foucault, 1969). Le mâle prime sur la femelle voire exclut la femelle. La chair (sexe) prime sur le verbe (genre).

Alice MILLIAT (1884-1957) fondera le Femina Sport, en 1911, et promouvra la pratique d'activités physiques féminines. Elle se heurtera ici à l'institué sportif et, notamment, au Baron Pierre Frédy DE COUBERTIN qui, au même moment, considère que :

«Les Jeux olympiques doivent être réservés aux hommes. Une Olympiade femelle serait inintéressante, inesthétique, et nous ne craignons pas d'ajouter : incorrecte, telle serait à notre avis cette demi Olympiade féminine. Ce n'est pas là notre conception de l'olympisme dans lequel [...] on doit continuer de chercher la réalisation de la formule que voici : l'exaltation solennelle et périodique de l'athlétisme mâle avec l'internationalisme pour base, la loyauté pour moyen, l'art pour cadre et l'applaudissement féminin pour récompense. » (DE COUBERTIN, 1912). ou encore que :

16 «Il va de soi que par son objet même la gymnastique utilitaire ne convient pas aux jeunes filles » (DE COUBERTIN, 1905).

Progressivement, alors que le nombre de filles pratiquant le sport s'accroît (par le biais notamment de patronages ou d'associations périscolaires), la résistance des fédérations sportives masculines devient de plus en plus violente et cela se passe dans plusieurs pays d'Europe simultanément. Par exemple, la Fédération anglaise de football - association (football actuel) interdit, en 1921, l'accès aux stades des femmes, car « le sport du ballon rond n'est pas décent ou gracieux pour la femme et qu'enfin il est trop brutal, trop dangereux pour elle » (Le Miroir des Sports, 22/12/1921, p. 329).

Cependant, on voit que si l'institution rejette les femmes, dans des espaces non instituées, les choses sont plus complexes. De facto les femmes pratiquent.

résistance à la scolarisation des femmes, dans l'enseignement secondaire est aussi vive. On trouvera dans la contribution d'Yveline FUMAT à ce numéro (p. 13) une vigoureuse citation du journal Le Gaulois, certes très à droite, sur le thème: «Des lycées de jeunes filles? Pourquoi pas des casernes de jeunes filles?» Jules Simon écrit, pour sa part :

21 «Je soutiens qu'il est parfaitement inutile d'enseigner la chimie pratique aux filles [...] Il faut en effet, pour se livrer fructueusement aux études philosophiques, des cerveaux d'hommes mûrs déjà préparés » (idem).

Si ce ministre de THIERS peut être considéré comme relativement conservateur, voire réactionnaire, on ne doit pas moins considérer qu'il résiste à la scolarisation des filles comme, ailleurs, on résiste à leur « sportivisation » et à leur éducation physique. 

cas de Jules FERRY qui, dans son discours à la Salle MOLIERE, en Avril 1880, affirme : «Celui qui tient la femme, celui-là tient tout, d'abord parce qu'il tient l'enfant, ensuite parce qu'il tient le mari» (FERRY, J., 1880). Il en ira de même dans le champ de l'Éducation Physique à la même époque, de nombreux auteurs prônent une gymnastique « eugénique ", pour les femmes de manière officieuse, tel Jean-Baptiste FONSSAGRIVES (1869). Certes, il est bien question d'une gymnastique pour les filles et non d'une gymnastique mixte, mais comme pour l'éducation, il s'agit finalement de l'un des premiers pas. Ce premier pas se construit cependant davantage sur les bases de la « nature » que sur celle de la culture.

«Sans gymnastique, je ne saurais trop répéter, pas d'éducation physique chez les filles, pas de formes régulières, pas de proportions heureuses, pas d'aptitude à une maternité efficace, pas de descendance robuste. C'est dire la gravité de cet intérêt.» (FONSSAGRIVES, 1869, p. 125).

Jusqu'aux années trente cette position eugéniste restera relativement majoritaire chez les médecins. Cela conduira à distinguer nettement pratiques physiques masculines et pratiques physiques féminines. L'un des discours les plus frappants de ce point de vue se situe d'ailleurs dans le Traité d'Education Physique de 1930, sous la plume de Marcel LABBE, le directeur de l'ouvrage :

"La femme est construite avant tout pour procréer ; par conséquent la culture du corps féminin est au moins aussi importante que celle du corps de l'homme, puisque les qualités physiques maternelles, outre qu'elle facilitent l'eugénétique, se transmettent à l'enfant. La fin suprême de la femme est le mieux-être de la postérité. En cultivant la valeur physique et physiologique de la femme, c'est toute la race qu'on améliore avec elle » (LABBE, 1930, p. 540).

27 Évidemment, il n'est pas question, là, de mixité même s'il est question d'éduquer physiquement les filles pour des raisons « eugénétiques».

Pourtant, il faut, comme le font remarquer le rapport du Sénat et un certain nombre d'auteurs (ZANCARINI-FOURNEL, 2005), distinguer le discours officiel et les pratiques réelles. Officiellement les filles et les garçons sont séparés. Officiellement, à la fin du XIXe siècle, les filles ne font pratiquement pas d'activités physiques. La réalité, comme on l'a $\mathrm{vu}$, peut-être toute autre et, pour ce qui concerne la mixité, cette réalité n'est pas aussi simple qu'il peut y paraître.

29 En effet, si les documents officiels de l'éducation physique, jusqu'en 1935, présentent partout des corps d'hommes (mais les enfants ne sont pas beaucoup plus présents que les femmes, à part dans le Manuel d'exercices physiques et de jeux scolaires de 1907 où, toutefois, ce sont toujours des garçons) en train de faire des exercices, on sait que les filles pratiquent et pratiqueront encore davantage par la suite (notamment avec Alice MILLIAT). Elles organisent d'ailleurs des "contre - Jeux Olympiques» ou des "Jeux Olympiques parallèles » en 1922 et 1926.

\section{La pratique vécue plus métisse que les textes}

30 À la fin de la première guerre mondiale, le paysage de l'éducation physique changera de manière très conséquente, $\mathrm{du}$ moins pour ce qui concerne son aspect officieux. Les 
femmes commencent à y trouver une place réelle, même si l'aspect officiel n'en est nullement bouleversé.

Bien entendu, cela doit être mis en rapport avec les combats féministes et, surtout, la place prise par les femmes dans le processus de production au cours de la première guerre mondiale. On ne peut pas non plus négliger le fait que le travail industriel « léger » qui se développe (bonneterie, filatures de soie ou de coton...) donne une place de plus en plus grande aux femmes de la classe ouvrière dans le processus productif. En tout état de cause, les suffragettes vont secouer la vieille Europe et, bien sûr la France.

Si en Suède le droit de vote des femmes est déjà partiel entre 1718 et 1771 puis en 1862, il devient total à partir de 1919. En Norvège, c'est en 1913, en Finlande, en 1906, au Danemark en 1915, en Russie, en Tchécoslovaquie, en Pologne et en Géorgie, en 1918. L'Allemagne, les Pays-Bas, le Luxembourg et la Belgique permettent cet accès citoyen en 1919.

33 En France les choses sont plus complexes, et c'est au bout d'une longue histoire de luttes, d'avancées et de reculs, que le droit de vote sera accordé aux femmes en 1944.

C'est au cours de la même période (1922) qu'est publié l'ouvrage de Victor MARGUERITTE La Garçonne. Monique LERBIER, qui en est l'héroïne porte des jupes courtes, ou le pantalon, coupe ses cheveux, fume, revendique fièrement son indépendance sexuelle. Bref, elle se réfère assez souvent à un stéréotype de genre masculin. Ce que l'on retrouvera, finalement, dans le comportement des premières sportives. Elles adoptent un comportement initié par les hommes (compétition sportive, dépassement de soi...). C'est en ce sens que Nicole MOSCONI parle, pour la mixité, d'un "masculin - neutre " (MOSCONI, N., 1989). En fait, le comportement androgyne se forme sur le stéréotype de genre masculin et non sur le stéréotype de genre féminin. Le sport fédéral en est un excellent indicateur tout comme l'éducation physique scolaire, on va le voir plus loin.

Pour la mixité, de facto, le rapport du Sénat, pointe bien que même si elle est interdite par un arrêté du 27 Brumaire an III, texte réitéré le 29 Février 1816, sous la Restauration, le fait que l'on ne crée pas d'écoles primaires de filles partout, et que l'on n'interdise pas aux filles d'être scolarisées, laisse penser que, dans les petites classes, il doit y en avoir de mixtes. Cela doit être le cas, notamment, dans les hameaux ou dans les plus petits villages qui sont très nombreux, dans une France essentiellement paysanne.

Le texte du Sénat, rappelle que, dans le département de la Manche, en 1863, il y a 216 écoles publiques mixtes, 203 qui ont installé une cloison entre les garçons et les filles et 201 qui ont une entrée commune. Autrement dit, il y a un énorme pas des textes à la pratique, puisque dans ce cas, qui semble assez répandu, un tiers des écoles est mixtes. Sous le second empire, en 1866, il y aurait eu 14756 écoles publiques mixtes dirigées par un homme, 1666 dirigées par une femme. En 1891, il y a 13700 écoles mixtes dirigées par un homme et 5500 dirigées par une femme. Autrement dit, la féminisation des directions d'école est en cours et la mixité n'a que peu régressé. 


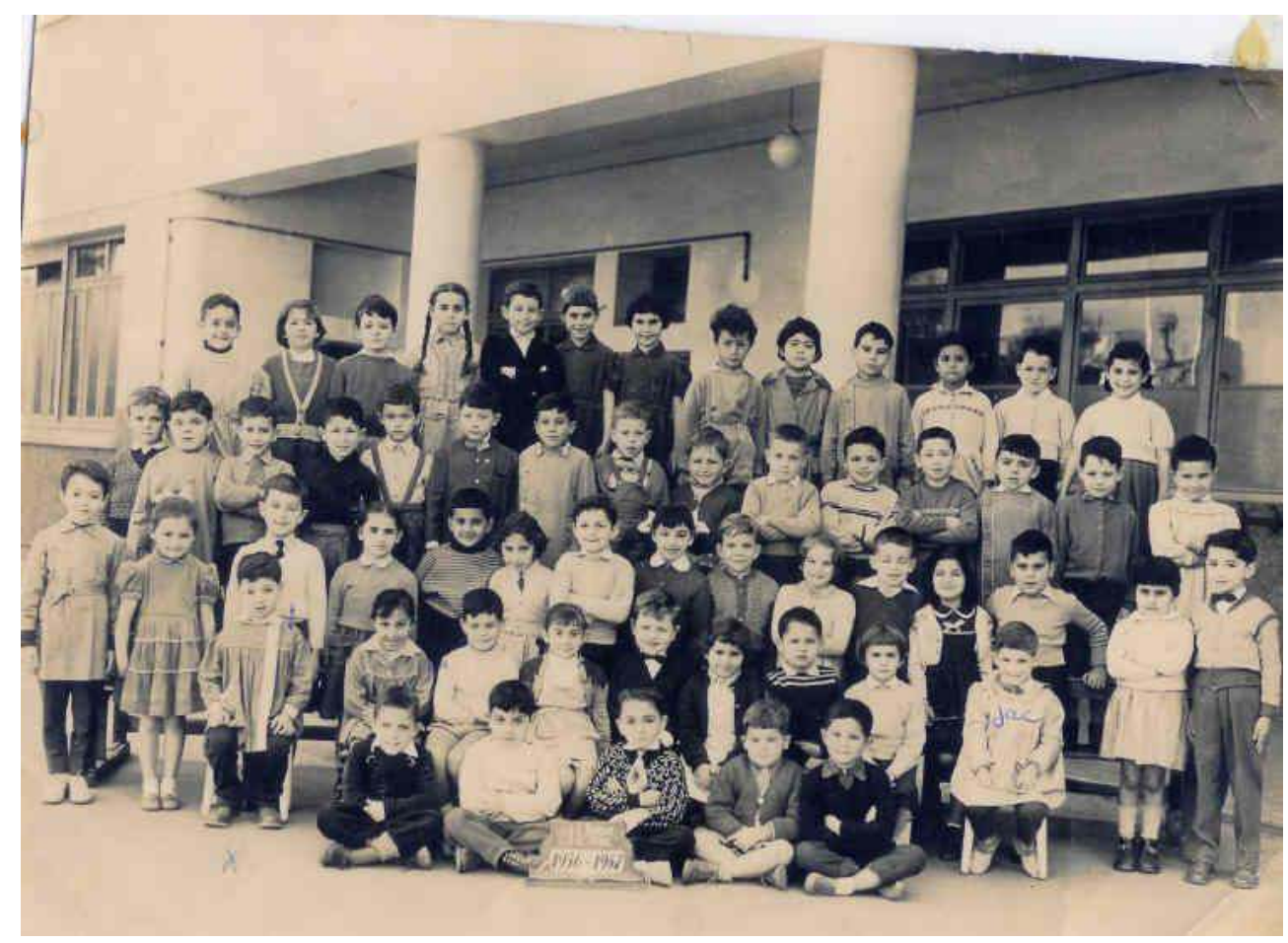

Classe mixte d'école maternelle, grande section, années 50

Si l'on observait les Collèges Royaux ou les Lycées Impériaux à la même époque, sans doute que la réalité serait très différente, mais le nombre des élèves (environ 80 000) y serait également bien plus restreint puisque la grande masse de ceux-ci (environ 3,8 millions) se trouve dans l'enseignement primaire et primaire supérieur.

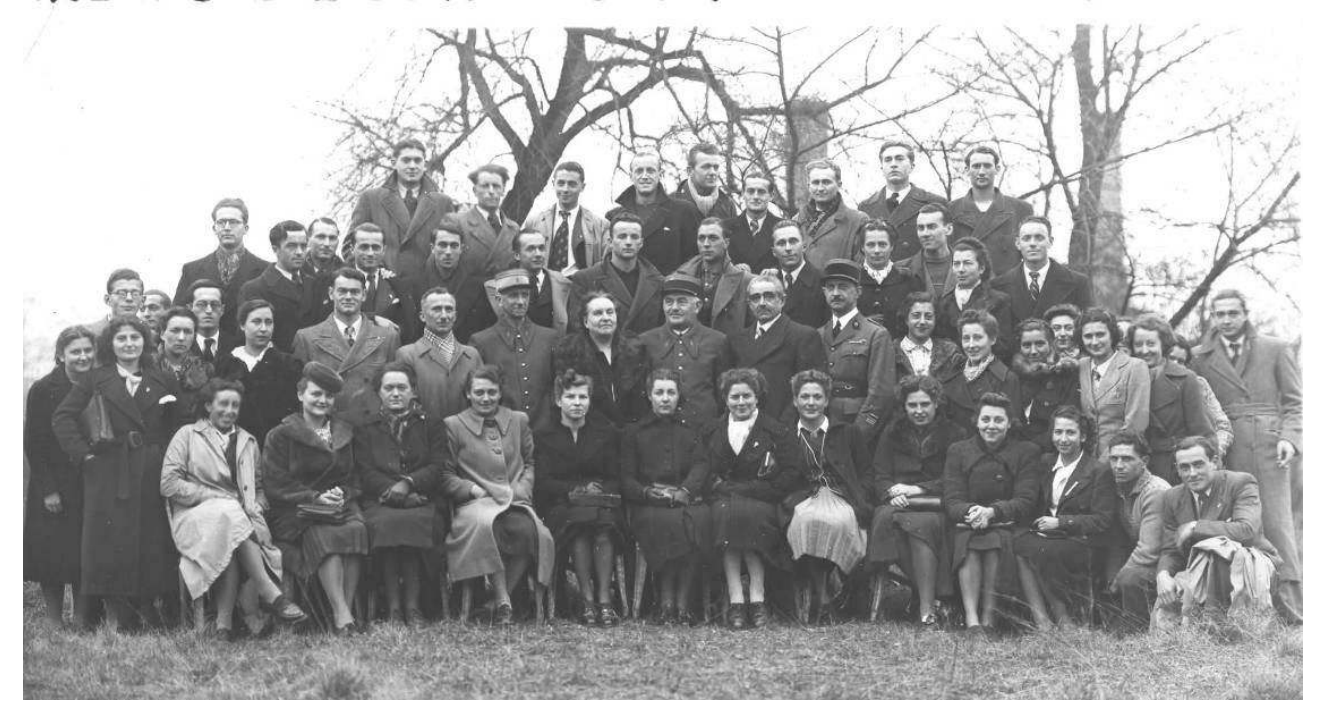

Promotion ENEP, mixte, 1939, autour du directeur, Ernest Loisel, au deuxième rang entre deux militaires. Celui-ci est agrégé d'Allemand. Photo dégagée de droits d'auteurs

La loi du 30 octobre 1886, loi Goblet, qui organise l'école primaire et surtout l'école primaire supérieure républicaine, ne pose plus la condition du démixage des classes et, ici encore, de facto les cours complémentaires et même, parfois, mais bien plus rarement, les écoles primaires supérieures, contrairement aux Écoles Normales, qui demeurent des sortes de lycées bis, seront souvent mixtes. Très probablement, les garçons et les filles 
étaient-ils co-instruits aussi bien en éducation physique que dans les autres disciplines au sein d'un seul cours complémentaire. Plusieurs témoignages d'anciens élèves de cours complémentaires (plus tardifs, vers les années 30-40), recueillis dans le cadre d'une recherche en cours, en témoignent (GLEYSE, J. 2009). Très souvent, il n'y a qu'un cours complémentaire ${ }^{1}$ dans une petite ville (entre 1000 et 5000 habitants) et celui-ci est donc mixte.

Même pour ce qui est des Écoles Normales, on voit sur la photographie ci-dessus, représentant les élèves de l'une des premières promotions de l'École Normale (supérieure) d'éducation physique (créée en 1933), que celle-ci est mixte, notamment en raison du nombre restreint de ses étudiants et étudiantes (une trentaine). Cependant, paradoxalement, en raison de pratiques très différentes au plan physique (sport pour les garçons et rythmique pour les filles) et à l'accroissement des effectifs, l'École Normale supérieure d'éducation physique sera séparée en une école de filles (Châtenay-Malabry) et une école de garçons (Vincennes), comme dans un mimétisme avec les autres écoles normales supérieures, en 1946. Les postes de Certificat d'aptitude d'éducation physique et sportive qui étaient mixtes jusqu'à la deuxième guerre mondiale, seront démixés à partir de 1950. Un peu comme si l'EPS suivait le mouvement inverse de l'école, à ce moment. Cela peut sans doute être attribué à sa sportivisation: le sport, dit Catherine Louveau (2000) est structuré sur un stéréotype masculin).

40 Par contre, les deux Écoles Normales Supérieures historiques, celle de Sèvres (29 Juillet 1889) pour les femmes (et celle d'Ulm: on peut dire an III) pour les hommes ne fusionneront, pour devenir mixtes, qu'en 1985.

41 Mais on ne peut supposer qu'il en va de même de l'enseignement supérieur à la même époque (à partir du moment où les filles peuvent s'y inscrire). Les Universités ouvrent certainement leurs portes aux filles. Tel est le cas, cette fois plus officiellement, de l'enseignement à l'école qui ne s'appelle pas encore «maternelle», mais "préélémentaire ».

Officiellement les Khâgnes deviennent mixtes en 1923 et des élèves filles sont autorisées à fréquenter les lycées de garçons (et l'inverse) dès 1922 (peut-être avant officieusement).

Comme on le sait, par ailleurs, les programmes du baccalauréat deviendront identiques pour les filles et les garçons en 1924, mais cela ne signifie pas que les classes de terminale sont mixtes. Au contraire, dans l'enseignement secondaire la plupart des établissements originellement catholiques (et c'est cela qui est important) et privés, sont démixés. Les filles, plus fréquemment, passent le brevet supérieur et non le bac qui ne leur est pas proposé comme diplôme de fin d'étude du secondaire. Très rares, comme on l'a vu, sont celles qui auront le baccalauréat avant cette date.

Il convient alors de faire remarquer que la gymnastique est présente au brevet supérieur (pour les garçons comme pour les filles), ce qui est loin d'être le cas au baccalauréat (elle devient facultative en 1941). On le voit clairement dans le document ci-dessous. Les filles qui poursuivent leurs études dans le primaire supérieur ou dans le secondaire, avant 1924, ont donc, peut-être, tout autant que les garçons, pratiqué la gymnastique. Celle-ci, de toute façon, ne sera obligatoire, à raison de deux heures par semaine, dans l'enseignement secondaire qu'à partir de 1925. Elle figure depuis 1882 au programme de l'enseignement primaire, mais il n'est pas du tout certain (ANDRIEU, 1990, GAY-LESCOT, 1999), dans les écoles rurales, qu'elle soit enseignée. Souvent les instituteurs et institutrices pensent que les travaux des champs (vendanges, moissons, ensilages...) et les 
jeux dans la campagne, les baignades en rivière ou étang, voire la chasse, la cueillette, etc., suffisent aux exercices physiques de leurs élèves.

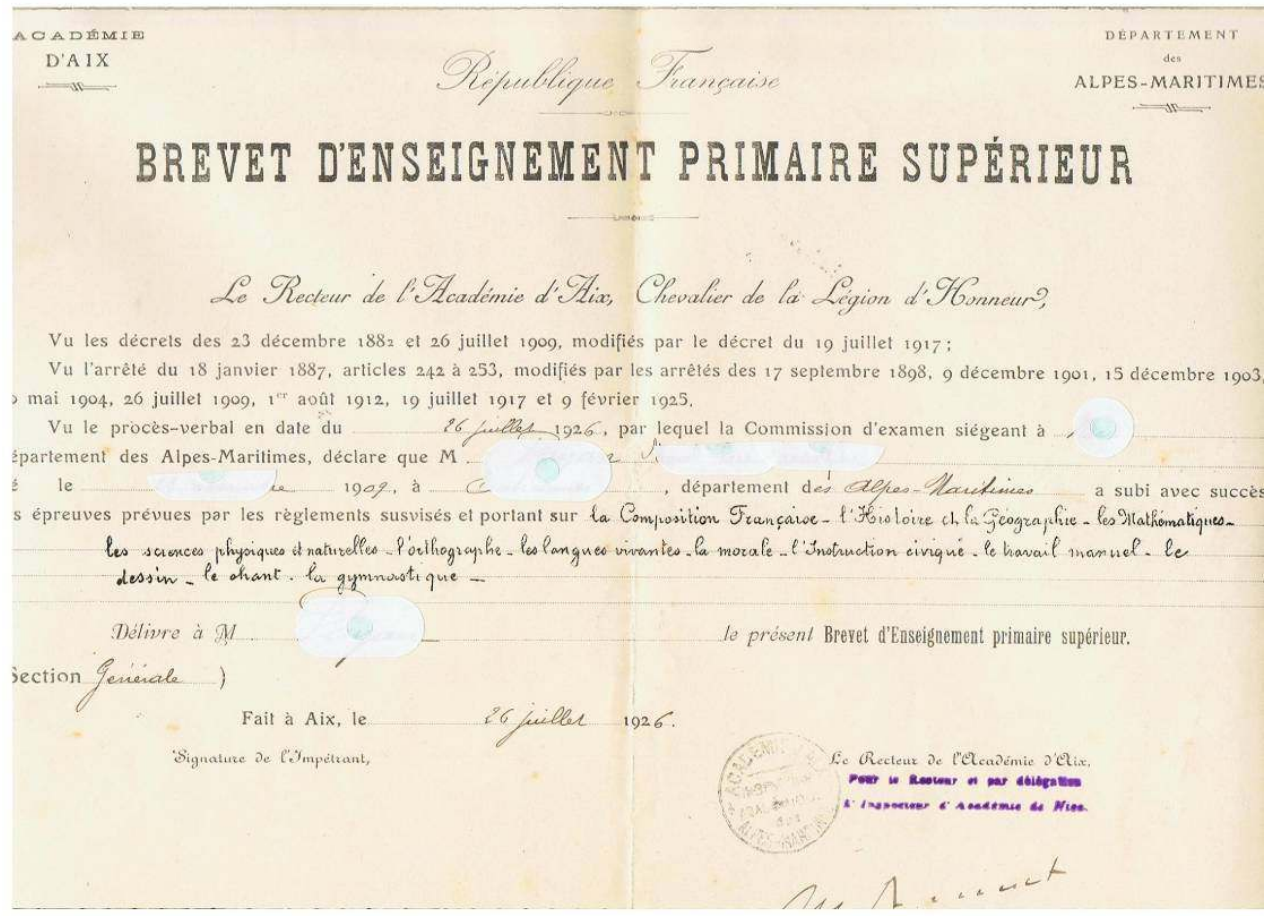

On voit sur ce document officiel de l'académie d'Aix-Marseille, de 1926, la gymnastique à la dernière place mais présente au programme du brevet supérieur qui clôt la formation en École Normale.

Sans vouloir aveuglément suivre le fonctionnement analogique du domaine des pratiques physiques (Gymnastique, Éducation Physique) et des écoles, on doit pourtant noter que les Jeux Olympiques deviendront mixtes en 1928. Par contre, les pratiques physiques proposées, aussi bien dans le monde du sport que dans le monde scolaire resteront très 
discriminées. Ainsi s'il y a apparence de mixité, les contenus implicites et explicites proposés aux filles et aux garçons sont très différents.

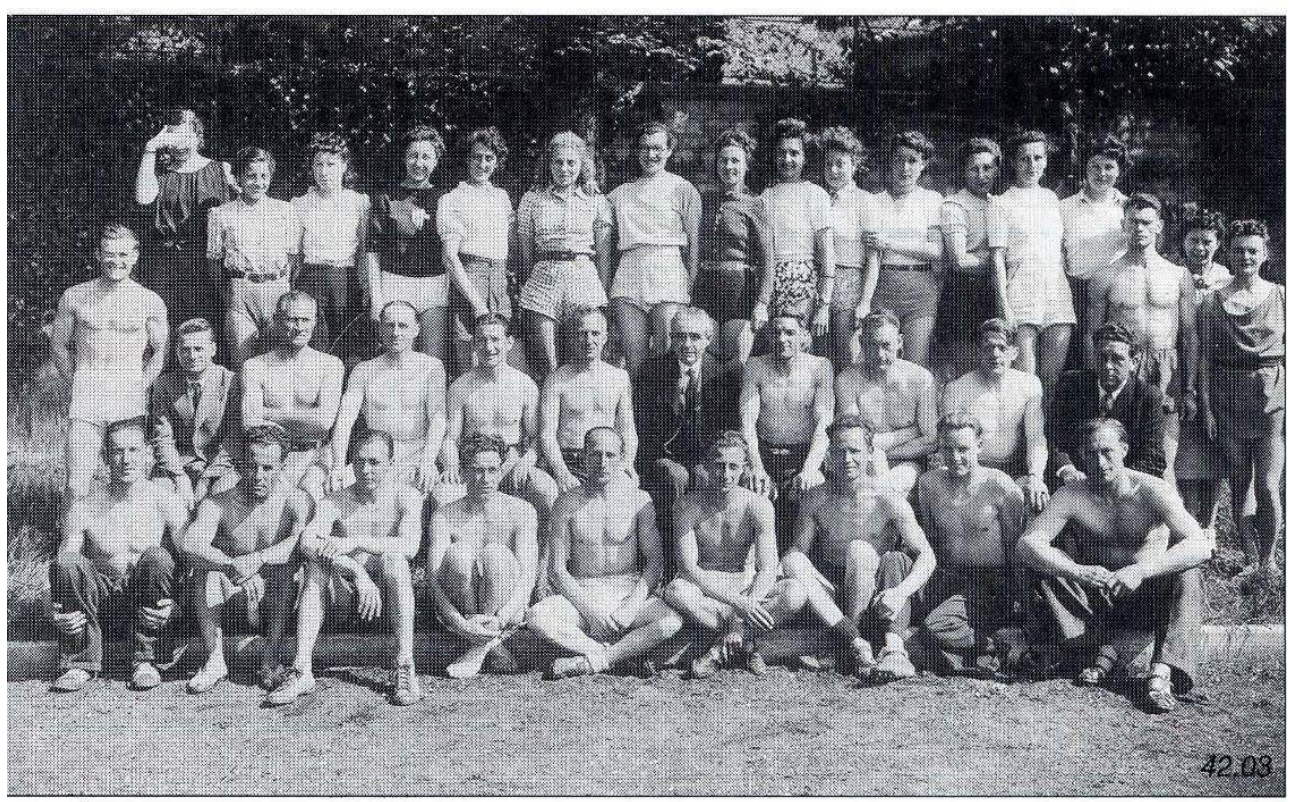

Promotion ENEP mixte, prise l'été, autour du directeur Ernest LOISEL, le seul à être en costume, 1936. Photo dégagée de droits.

Une étude biographique, réalisée en 2004 par Luc ROBENE, intitulée : Itinéraire d'une " prof de gym ». Paulette MORISSON (1914-2000) (ROBENE, 2005, p. 319-337), montre que la mixité est une réalité vécue pour cette sessionnaire d'une des premières promotions de l'Institut régional d'éducation physique de Bordeaux, puis de l'École Normale d'éducation physique (en fait École Normale supérieure) de Paris, en 1938, qui a fréquenté l'école primaire supérieure et obtenu son Brevet Supérieur, en 1933, à l'âge de 19 ans. Elle explique très clairement, à son interviewer, que la totalité des cours intellectuels est mixte (co-instruction), à l'IREP de Bordeaux et même certaines activités physiques (ce qui est très peu visible à l'époque dans le domaine sportif fédéral) dont les effectifs nécessitent un nombre suffisant de participants. Tel est le cas du handball pratiqué à 11, en plein air, à ce moment-là. En réalité, ce qui justifie la mixité dans plusieurs pratiques c'est le nombre restreint de cessionnaires : 9 filles et 11 garçons. Si cela se produit à l'IREP de Bordeaux on peut imaginer que c'est aussi le cas dans les autres IREP de France qui sont créés, pour la plupart, entre 1927 et 1933 et scolarisent parfois moins d'étudiants.

L'interviewée, pourtant, ne se leurre pas sur la domination masculine et le fait que ce sont toujours les garçons qui sont mis en avant comme les seuls véritables "sportifs", ce qu'elle n'apprécie pas du tout, car elle considère ses performances aussi respectables que celles des garçons. Comme cela a été souligné, sa promotion de l'ENEP sera également mixte.

Bref, si la mixité ne touche sans doute que très peu les collèges et les lycées et minoritairement les classes de primaire, il n'en va probablement pas de même de la mixité à l'université, dans les cours complémentaires et dans les plus petits établissements (un témoignage d'Adrienne DURAND-TULLOU, 1989, pour un hameau: Rogues sur le Causse de Blandas, montre la mixité de sa classe de primaire à plusieurs niveaux, en 1938). Cependant, comme dans le monde du sport et de l'éducation physique le modèle masculin y reste sans doute très dominant au plan symbolique et structurel. En 
cela, il ne s'agit pas d'un métissage qui grandit les deux parties mais, de manière plus probante, d'une «andro - colonisation» de l'espace scolaire et de celui des pratiques physiques.

\section{Mise en place d'une mixité « masculin - neutre » ou d'un féminin « masculin atténué ». Métissage ou colonisation?}

Afin de mesurer combien l'entrée dans la mixité se réalise sur la base d'un "masculin neutre » (MOSCONI, 1989) ou d'un « masculin atténué » (ARNAUD, 1996) et non sur la base d'un métissage, d'une "androgynisation", à part chez quelques très rares auteurs, il convient de relire certains textes concernant l'éducation physique, aussi bien dans les textes officiels que dans les manuels scolaires. Il suffit aussi d'observer les images des manuels scolaires, par exemple d'hygiène ou de morale qui, parfois, comportent des chapitres éducation physique et des photographies ou des dessins au trait. En règle générale, si l'éducation physique y est valorisée aussi bien pour les filles que pour les garçons, il ne s'agit pas du tout d'y pratiquer les mêmes activités physiques. Le sport reste très longtemps proscrit pour les filles. Par exemple, un Manuel de Morale de 1935, pose clairement pour les filles que :

"Ici comme en toutes choses il faut savoir mesure garder. Il est bon d'éviter les entraînements excessifs [...] le corps ne doit pas être une guenille. Ce n'est pas une raison pour en faire un tyran » (AB DER HALDEN, C., 1935, p. 19).

Ci-dessous, on peut voir, dans un manuel d'hygiène destiné aux classes de 3ème, de 1926, que la force et l'énergie sont valorisées chez les garçons alors que la grâce et l'esthétique sont mises en exergue pour les filles. On voit aussi que filles et garçons sont séparés et ne pratiquent pas ensemble. 


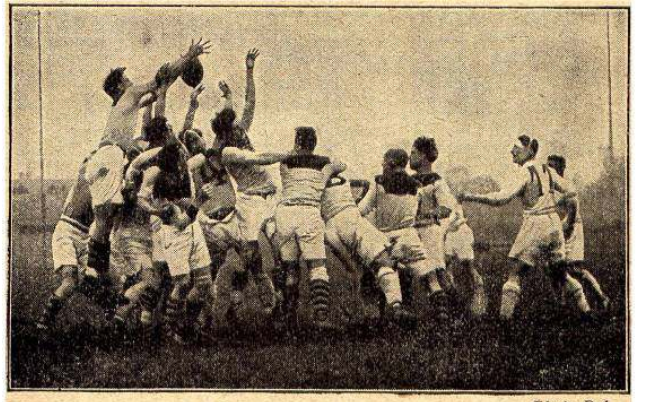

Fig. 236. - Le foot-ball.

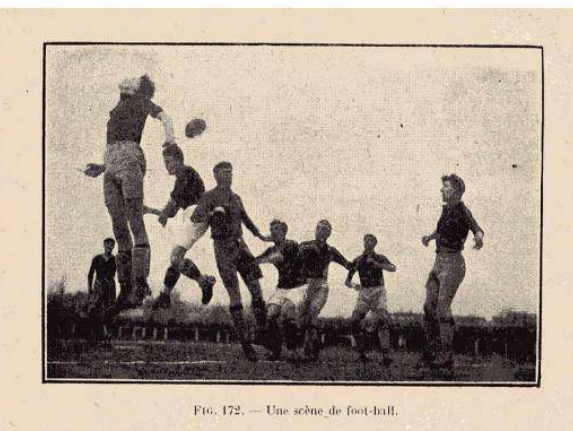

Fit. 172. - Une sine de frim t.ral

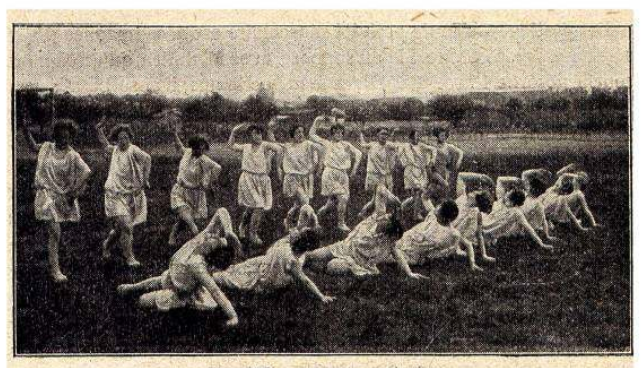

Fig. 225. - Danses rythmées.

Illustrations du point concernant la gymnastique d'un manuel d'hygiène de 1926 (Bouchet, 1926).

Autrement dit, même si les deux doivent bénéficier d'une activité physique, il n'est pas question que cette activité physique soit identique pour les deux sexes. Par ailleurs, si l'on observe les photos, on constate aussi que les hommes sont représentés dans une " élévation ", une ascension et les femmes, au contraire, dans une forme de soumission et de descente au sol. Cela est peut-être simplement anecdotique, pourtant Catherine LOUVEAU (2000) a bien repéré, notamment dans les représentations iconographiques médiatiques du sport lors des Jeux Olympiques que, la plupart du temps, les stéréotypes de "genres " journalistiques s'y expriment à plein. Ainsi, les femmes seront beaucoup moins représentées que les hommes dans des postures agressives ou dominantes, mais plus fréquemment, dans des attitudes de séduction, souriante, esthétisées. Tel est le cas, également, des représentations iconographiques des manuels scolaires. L'éducation physique est, en ce sens un bon indicateur de la mise en place de la mixité comme « masculin - neutre » ou comme « masculin atténué » pour les filles. 

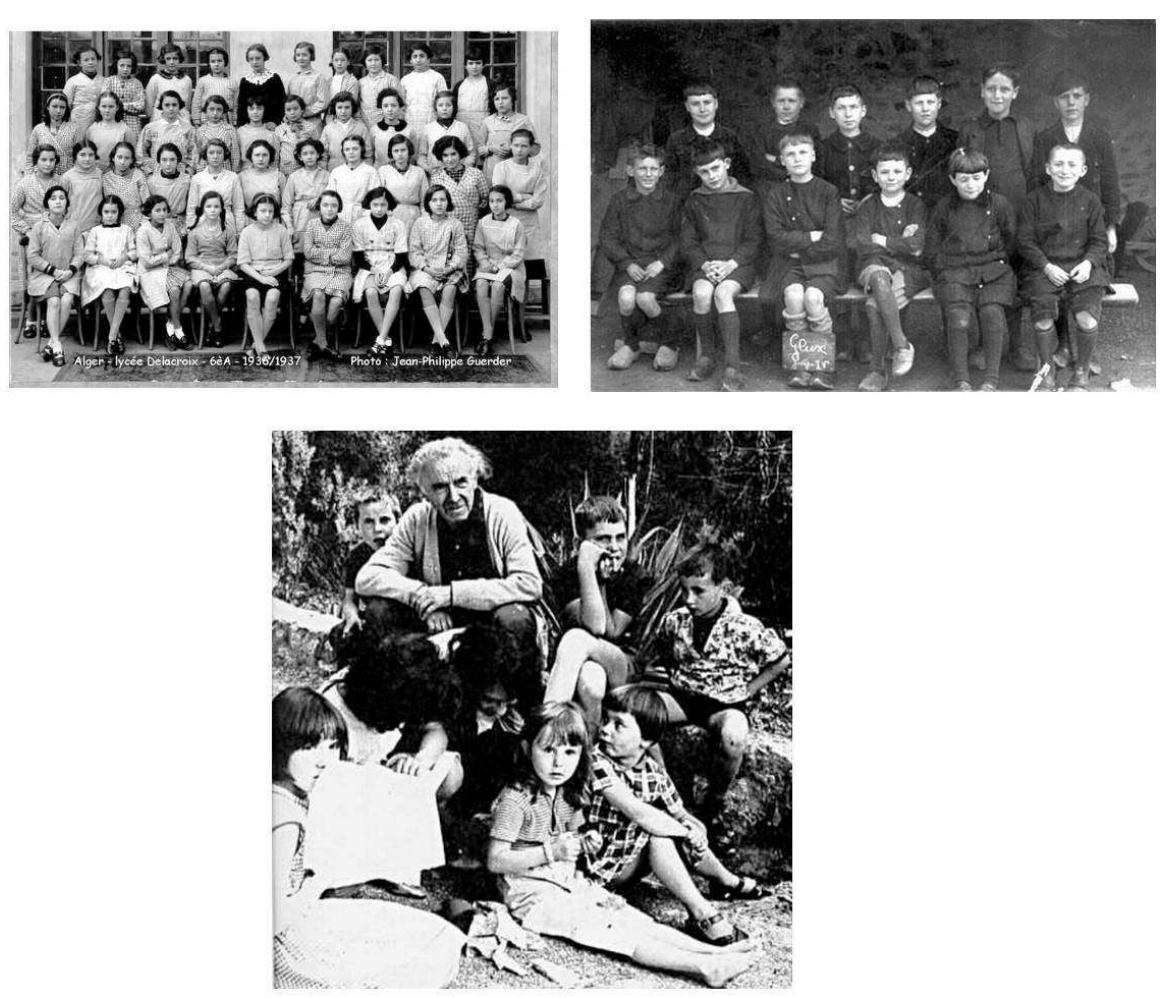

Classes primaires des années 1930. Deux classes démixées. On reconnaît en bas le groupe mixte de Célestin FREINET. Photos libres de droits

On notera que cette vision critique était celle de Simone de Beauvoir dans Le Deuxième sexe (1949), puisqu'elle y expliquait très clairement :

«On ne nait pas femme : on le devient ... C'est l'ensemble de la civilisation qui élabore ce produit intermédiaire entre le mâle et le castrat qu'on qualifie de féminin. »

Ce «masculin neutre» s'exprime très clairement, au cours de la deuxième guerre mondiale, et bien après celle-ci dans le domaine de l'EPS, mais très certainement aussi, dans d'autres secteurs de l'enseignement et dans de multiples disciplines scolaires. Dans l'iconographie de L'Illustration, page suivante, on voit explicitement (cela n'est pas très surprenant sous le gouvernement de PETAIN et de Vichy) que l'homme est mis en valeur, au premier plan, et que la femme, qui pourtant pratique la même activité (le javelot), n'est visible qu'en arrière plan. De nombreuses représentations iconographiques en EPS, au cours de la même période et jusqu'aux années quatre-vingts, fonctionnent sur ce stéréotype. La femme est, en quelque sorte, un homme en moins, qu'il y ait mixité ou non. 


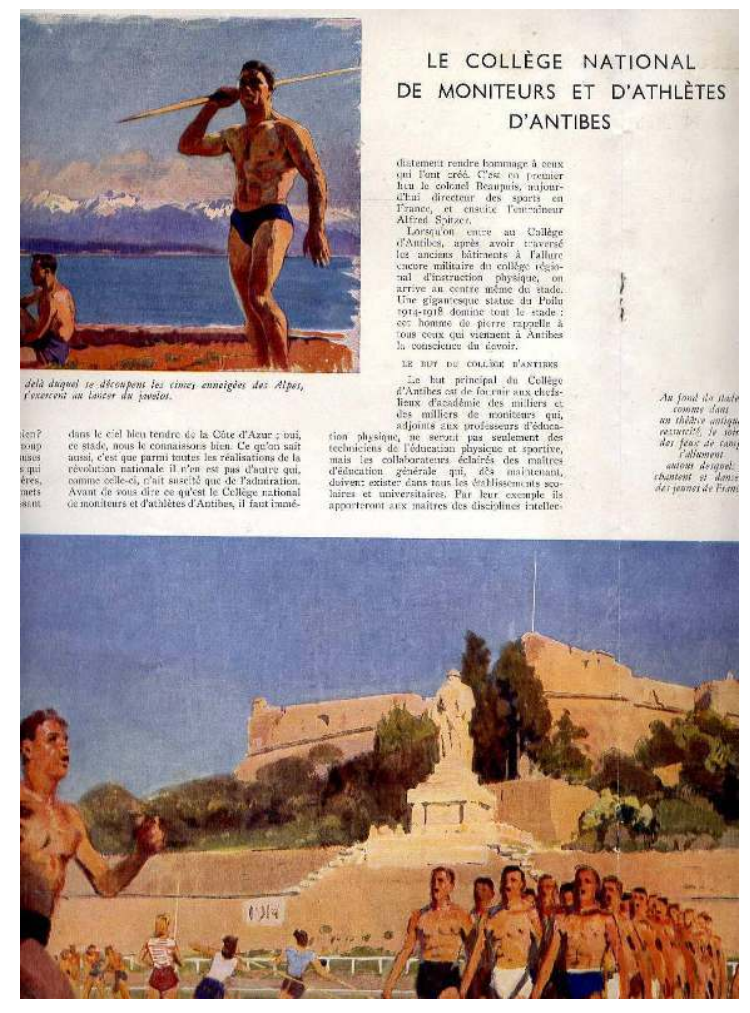

L'Illustration, 8 Août 1942, photo libre de droits. cette vision de la femme sous-homme. Ainsi Georges DEMENIJ, dans plusieurs de ses ouvrages constate avec regret que : «Les procédés d'éducation doivent être adaptés aux sexes; les habitudes et les préjugés le veulent ainsi » (DEMENIJ, 1902, p. 37). Georges HEBERT va plus loin en affirmant que sa méthode « s'applique aussi bien à l'éducation des filles que des garçons » (HEBERT, 1936, p. 9) et en affirmant que «la femme en un mot peut devenir une athlétesse » (idem). Plus clairement encore, bien qu'ayant publié un ouvrage intitulé: Muscle et beauté plastique féminine, en 1919, il précise, regrettant également les stéréotypes sociaux :

"La jeune fille qui serait développée et entrainée d'une façon aussi complète et poussée que le jeune homme pourrait se mesurer à peu près à égalité avec lui dans l'ensemble des épreuves. Mais le rôle social et familial de la femme, les habitudes de vie, la façon dont on l'élève, sa mentalité, ses aspirations, dont il faut bien tenir compte, entravent son développement et ne la disposent pas en faveur des manifestations de force » (HEBERT, 1911, p. 106).

Pour cela, il sera vivement critiqué par des auteurs plus conformistes ou moins féministes, voire conservateurs, plusieurs années plus tard et jusqu'aux années cinquante encore :

"Toute l'action d'HEBERT tend à faire un homme et, ainsi que le disait MONTAIGNE, à lui roidir l'âme et le corps. Chez la femme il développe les mêmes qualités et tend au type de la femme virile » (CAMBIER, in LABBE, 1930, p. 479).

Dans les ouvrages que Georges HEBERT publie, à partir de 1909, où les photos sont pléthores, les femmes sont tout autant mises en valeur que les enfants (ce qui est très rare ailleurs). On voit même dans L'éducation physique virile et morale par la méthode naturelle 
(1936) plusieurs images de cours «mixtes », ou du moins de co-instruction, y compris près d'un bassin de natation.

61 En 1946, un cahier de texte de 3ème année de cours complémentaire - corroboré par un témoignage recueilli par un entretien affirmant que les cours de gymnastique étaient mixtes, mais que les garçons et les filles ne faisaient pas nécessairement les mêmes exercices - montre que les cours de gymnastique sont bien présents à l'emploi du temps.
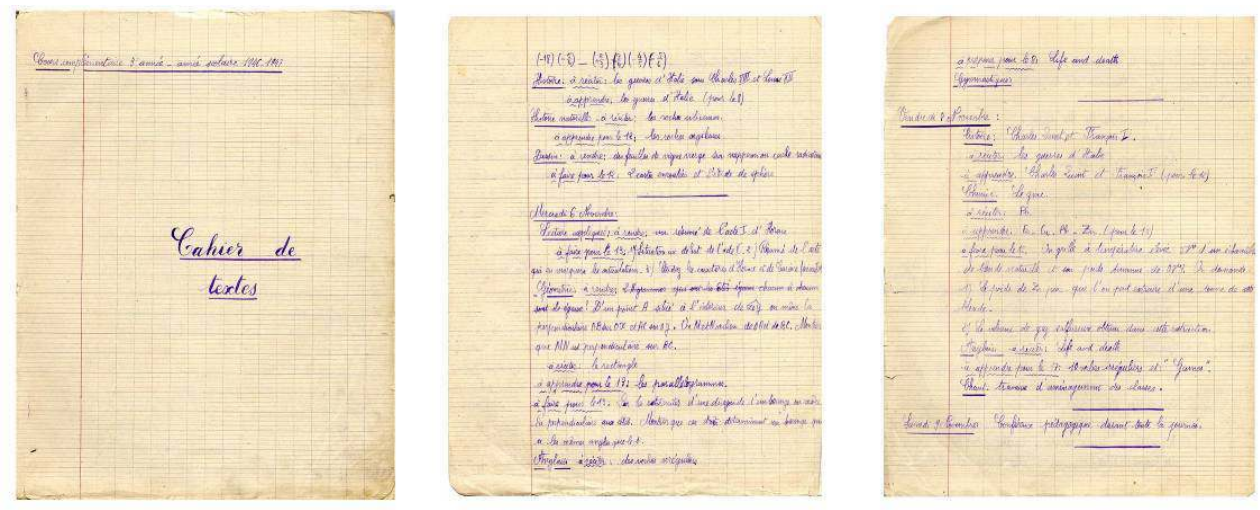

Cahier de texte de cours complémentaire 3ème année, année 1946-1947. À la date du mercredi 6 novembre figure la gymnastique $(2 \mathrm{~h}$ ) dans ce cours décrit comme mixte par la titulaire du cahier

Encore une fois, selon le rapport du Sénat (2004), mais cela a été validé par de multiples historiens de l'éducation (PROST, 1992, LELIEVRE, 1990, BAUDELOT \& ESTABLET, 1992 ...), la mixité officieuse puis officielle s'installe dans le système éducatif français, et donc sans doute aussi en Education Physique, sans que l'on y prenne garde.

«Au cours de l'année scolaire 1958-1959, on compte, selon des chiffres fournis par le ministre de la jeunesse, de l'éducation nationale et de la recherche à votre rapporteur, 21861 écoles mixtes sur 73059 écoles primaires, soit à peine $30 \% .25623$ écoles sont réservées aux garçons, et 25.575 aux filles ».

En 1965, la mixité est étendue à toutes les écoles élémentaires nouvellement créées, par la circulaire du 15 juin 1965. Des textes de 1962 et 1968 renforcent les pouvoirs des recteurs en matière de fusion des écoles de garçons et de filles (sans doute, en partie, pour des raisons économiques). À la fin des années 1960, presque toutes les écoles primaires sont mixtes. En 1967-1968, les classes distinctes de garçons et de filles ne subsistent que dans les grosses écoles.

En application du décret du 3 août 1963, les collèges d'enseignement secondaire (CES) sont mixtes dès leur création (réforme dite "CAPELLE-FOUCHET»), pour faire face à l'explosion des effectifs scolaires, résultant elle-même, de la croissance démographique c'est l'époque du baby boom - et d'une demande sociale accrue en faveur de la scolarisation.

La mixité s'étend dans les lycées, au cours des années 1970. Les décrets d'application du 28 décembre 1976 de la loi dite «HABY », du 11 juillet 1975 rendent la mixité obligatoire dans l'enseignement primaire et secondaire.

«Aujourd'hui, les effectifs d'élèves, concernés par la non - mixité sont très faibles. Plus aucun établissement public ne fonctionne de façon séparée, à l'exception notable des maisons d'éducation de jeunes filles de la Légion d'honneur, soit environ 1000 élèves, et il n'existe qu'environ 200 établissements non mixtes dans l'enseignement privé, très souvent hors contrat. » (Sénat, 2003). 


\section{L'éducation physique indicatrice ou dissonante?} $\mathrm{du}$ fait que ne comptent que les points au dessus de la moyenne (autrement dit que l'on ne peut pas être pénalisé pour des points au dessous de la moyenne) au deuxième groupe d'épreuve du bac. Le taux de dispense des filles augmente en effet, de près de $50 \%$ alors que celui des garçons baisse. On peut voir là une inégalité dissimulée- ce que l'on appelle des "programmes cachés d'éducation" (hide curriculum) - très clairement mise en évidence. Et si l'on admet que l'éducation physique et sportive est un bon indicateur, de l'ensemble du système éducatif, on peut supposer que le reste du système fonctionne également sur ces programmes cachés (ce qui maintenant a été démontré par de très nombreux auteurs, dont Nicole MOSCONI, avec la loi des 2 / 3, 1 / 3 par exemple dans les interactions orales dans les classes mixtes de tous niveaux).

73 À l'inverse, il est possible de supposer que l'éducation physique et sportive devient dissonante, par rapport au reste du système éducatif, dans la mesure où la gestion de ses enseignants n'appartient plus à l'Éducation nationale, entre 1959 et 1982. Ceci pourrait expliquer pourquoi, paradoxalement, alors que le système éducatif français devient officiellement mixte, les pratiques physiques sont de plus en plus démixées, dans ce domaine. En fait, peut-être est-ce la pensée de l'institution (DOUGLAS, 1986, 1999) 
sportive qui s'applique à ce champ et non celle de l'Éducation nationale qui, visant des « élèves » et non des enfants, réalise en quelque sorte un « déni » du sexe et du genre.

Pourtant, au cours de cette période, de nombreux auteurs, en EPS tels Jean LE BOULCH (1966, 1971 et 1977), Pierre PARLEBAS (1967, 1985), Jean-Marie BROHM (1975) combattront la mise en forme sportive de l'EPS et, d'ailleurs parfois, sur la base des stéréotypes de genre (comme la revue Quel Corps ?).

Le journal édité par le SGEN CFDT, à ce moment (1977-1985), Le Corps Enchaîné milite pour une véritable mixité en EPS tout comme le journal de l'École Émancipée (SNEP, EPS) : Le Chrono enrayé, ou encore les bulletins du SNEEPS (maîtres et professeurs - adjoints d'EPS), mais à l'inverse, le Bulletin du SNEP, majoritaire pour les enseignants certifiés d'EPS, continue d'exiger le démixage et de demander que les postes hommes et les postes femmes soient différenciés. Les deux premières publications rejettent la mise en forme sportive de l'EPS, notamment pour des questions de stéréotypes de genre. Ils prônent pour cela des pratiques davantage ludiques ou d'autres formes d'activités physiques que le sport (par exemple l'Expression corporelle). C'est grâce à leur action que lors de la venue de la gauche au pouvoir, en 1981, la gestion des enseignants d'EPS retournera à l'Éducation nationale et, surtout, que celle-ci deviendra réellement mixte (concours du CAPEPS) à partir de 1988 (le concours du CAPEPS n'est plus démixé comme il l'était depuis la deuxième guerre mondiale).

76 Si l'on y réfléchit, on peut penser que c'est la même logique qui fonctionne à ce moment (dans les années 1970 à 2000 environ) pour les filières scientifiques et technologiques dans le système éducatif français. Les filles optent majoritairement pour les filières littéraires et de sciences humaines alors que les garçons se dirigent vers des filières scientifiques et technologiques. Or, la sélection pour les carrières les plus socialement porteuses s'effectue sur la base des mathématiques et des sciences. Il s'agit, en définitive et sans trop vouloir filer l'analogie, du même processus que celui qui est mis en œuvre avec le sport en EPS. Ce sont les pratiques valorisées par les hommes et les garçons qui servent de gradient de sélection et non celle valorisées par les filles. Les filles doivent donc se soumettre au modèle sportif masculin, comme elles doivent se soumettre au stéréotype scientifique qui ne leur convient pas mieux. À l'inverse, on pourrait aussi dire que les garçons, qui ne souhaitent pas entrer, ou qui n'entrent pas dans ces stéréotypes, seront dévalorisés (féminisés).

77 En EPS, les textes officiels et les pratiques, à partir des années soixante, contrairement aux pratiques d'éducation physique antérieures comme la gymnastique néo-suédoise ou la méthode naturelle, discriminent très nettement les filles comme inférieures (on se rappelle que Georges Hébert, propagateur de la méthode naturelle jusqu'à la fin des années cinquante, ne souhaite pas discriminer les filles, au contraire).

« En ce qui concerne les jeunes filles, cette orientation athlétique s'adressera plutôt à celles d'entre elles qui marquent pour le sport un intérêt ou des aptitudes [...] Pour les autres, les plus nombreuses, la part des leçons ordinairement réservée à l'initiation sportive s'efforcera de répondre à leur goût de l'esthétique et de l'expression corporelle » (Instructions du 21 août 1962).

Or, comme l'évaluation au Brevet des Collèges et au Baccalauréat devient essentiellement sportive, aussi bien pour les filles que pour les garçons, on ne peut qu'en déduire un désavantage conséquent pour les filles. Pourtant, à ce moment l'ensemble du système 
éducatif français est en train de devenir officiellement mixte, à part, sans doute, dans les sections technologiques où, comme en EPS, les stéréotypes genrés sont très forts.

Les Instructions Officielles du 19 octobre 1967 ne dérogent pas à cette logique: l'expression corporelle est globalement destinée aux filles et les sports de combats aux garçons. La gymnastique prend une part plus importante pour les filles que pour les garçons. Mais, encore une fois, ce sont les pratiques sportives qui sont au cœur des dispositifs d'évaluation au baccalauréat et au brevet des collèges. En conséquence de quoi les filles obtiennent de moins bonnes notes que les garçons.

81 En 1982, la situation change radicalement puisque, à la suite de l'élection de François Mitterrand et de l'arrivée de la gauche au pouvoir, en mai 1981, la gestion des enseignants d'EPS est à nouveau confiée à l'Éducation Nationale. En 1983, l'éducation physique et sportive devient coefficient 1 au baccalauréat, au premier groupe d'épreuves, autrement dit les points au dessous de la moyenne comptent également. Les dispenses d'EPS sont supprimées et se transforment en « exemption temporaire de pratique ». Cela signifie que l'EPS devient une matière de sélection (même si c'est à un faible niveau) comme les autres.

82 Le ministère veut alors vérifier si la mixité dans ce domaine est une réalité ou, du moins, quelle est sa situation de facto. En effet, la plupart des acteurs importants du moment savent très bien que beaucoup de classes en EPS, en raison essentiellement de la valorisation de pratiques sportives, sont démixées.

À titre anecdotique, j'étais alors enseignant d'EPS en collège, favorable à la mixité intégrale et partisan de la modification des contenus afin de diminuer le poids des stéréotypes masculins, et je me souviens très bien des difficultés qu'il y avait à avoir des classes mixtes. La plupart du temps, deux classes de même niveau étaient réunies et les garçons allaient avec un professeur homme et les filles, des deux classes, avec un professeur femme. Rompre cette logique supposait beaucoup de volonté pour faire changer les visions des collègues et de l'administration qui, généralement, s'opposaient à la mixité. Il était encore plus difficile de faire admettre que les contenus sportifs étaient « stigmatisant » pour les filles. 


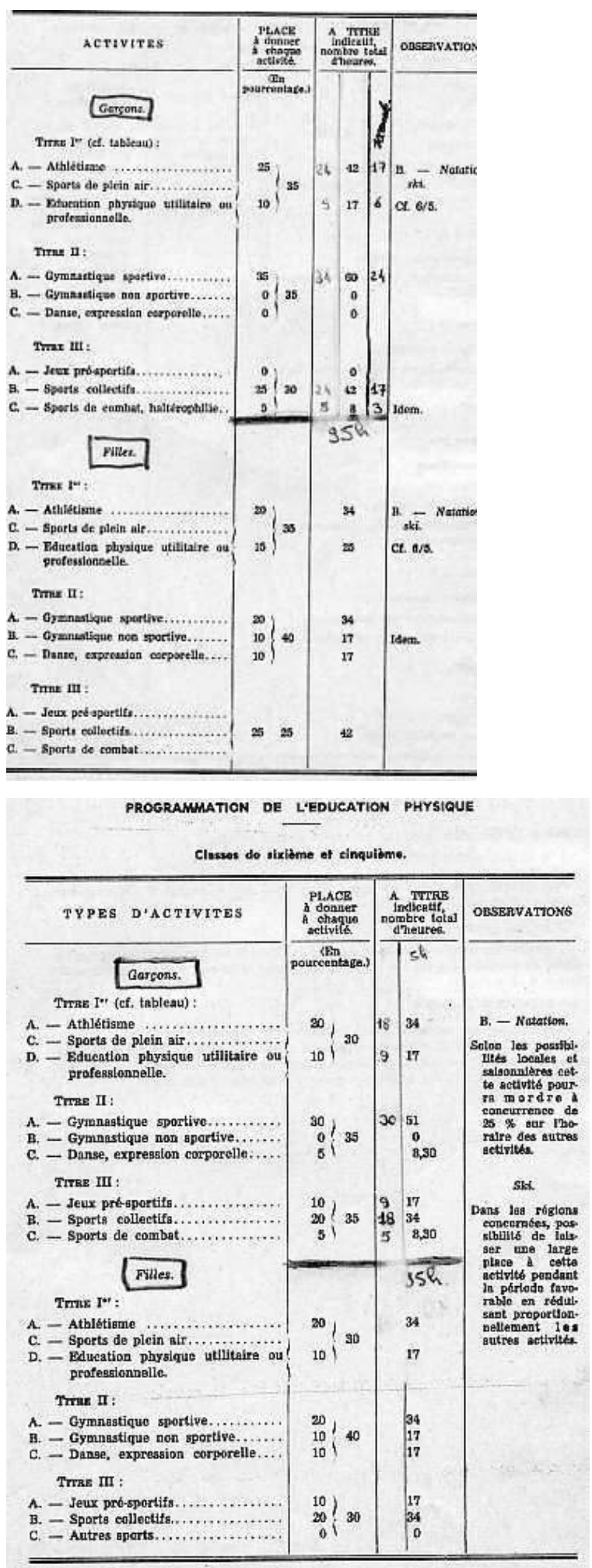

Exemples de tableaux demixés de la programmation de 1967 où l'on voit tout le poids des stéréotypes de genre dans les activités massivement sportives.

Une enquête mandatée par le ministère, en 1984, sous la direction de Michel VOLONDAT, montre, pour l'EPS, un tableau stupéfiant, dans un système éducatif officiellement devenu mixte depuis huit ans (décrets d'application de la loi HABY). L'EPS qui s'est développée sous la logique du système sportif (Ministère de la Jeunesse et des Sports) n'est 
globalement pas mixte. Seuls $21 \%$ des enseignants n'ont que des classes mixtes, $42 \%$ ne travaillent jamais en mixité, $21 \%$ font des groupes de niveaux qui conduisent le plus souvent à un démixage. Pourtant, au même moment les élèves, également enquêtés, sont très majoritairement favorables à la mixité $(56,8 \%)$ et ce d'autant plus qu'ils sont âgés (presque $80 \%$ en Terminale). Inversement et paradoxalement, les enseignants démixent d'autant plus les classes que les élèves sont âgés.
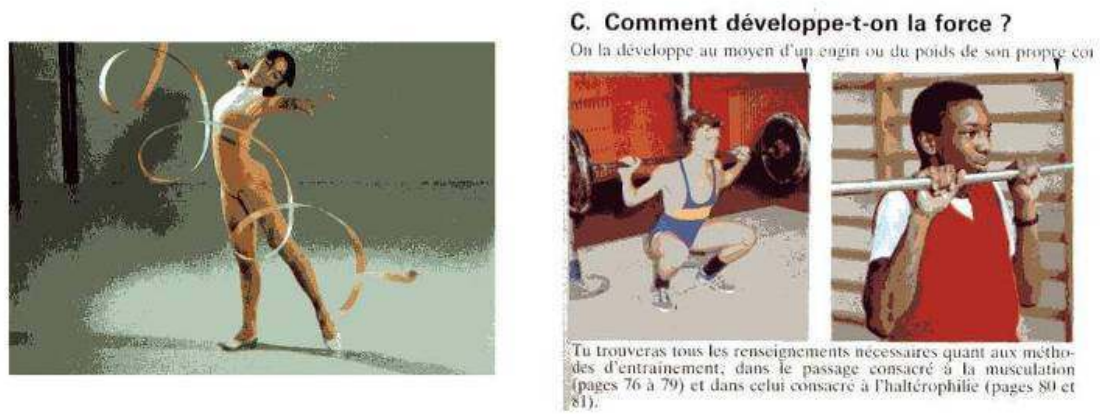

Images d'un manuel d'éducation physique paru en 1983 et destiné aux classes de 4e et 3e qui montre les stéréotypes de genre véhiculés encore à ce moment de manière très nette. certainement moins marquée, en ce qui concerne le reste de l'Éducation nationale, puisque les sections littéraires accueillent massivement des filles et les sections scientifiques et technologiques massivement les garçons. La différence réside dans le fait que ce ne sont pas les enseignants, comme en EPS, qui mettent en œuvre cette discrimination sexuelle, mais des "programmes cachés » d'éducation (et sans doute les enseignants qui construisent ces programmes cachés tout autant que les stéréotypes sociaux, les manuels scolaires ...). En définitive, ce qui est implicite et occulté pour le reste du système scolaire est très explicite et visible pour l'éducation physique, sportive.

pour cette raison que les instructions officielles de novembre 1985 - alors que le ministère, s'appuyant sur les positions du SGEN CFDT, dont il se sentait proche, souhaitait valider et imposer la mixité pour tous les élèves - devant la situation observée par l'enquête, se contentent d'une formule pour le moins ambiguë :

Le professeur peut constituer dans la perspective d'une pédagogie différenciée, des groupes de travail homogènes ou hétérogènes selon les moments de son enseignement. La même remarque vaut pour la mixité » (Instructions de 1985, signées Jean-Pierre CHEVENEMENT).

Il faut dire qu'à cette date aussi bien les concours de professeurs - adjoints (précédemment maîtres) d'EPS que celui de professeur certifié sont démixés. Seule l'agrégation externe, créée en 1983, est mixte (il n'y aura que 4 femmes sur 30 reçus pour la première promotion). Il faudra attendre que le SGEN CFDT dépose un recours devant la Cour européenne des droits de l'Homme et qu'une directive européenne interdise les discriminations sexuelles à l'emploi pour que le CAPEPS devienne mixte, comme tous les CAPES, en 1988. Toutefois, les pratiques physiques, massivement sportives, restent le plus souvent démixées, voire ségrégées. Cela est le cas, également, dans les lycées d'enseignement professionnels, pour de nombreuses sections, aujourd'hui encore, même si les établissements sont mixtes. 


\section{Mixité et programmes cachés d'éducation (hide curriculum)}

L'éducation physique et sportive reste encore aujourd'hui un excellent indicateur des rapports à la mixité de l'Éducation nationale, dans la mesure où « le corps est le symbole dont use une société pour parler de ses fantasmes" (Bernard, 1974, p. 141) et où les techniques du corps sont largement sous-tendues par les rapports de genre.

Comme on vient de le voir, à partir de 1988, les postes hommes et femmes en EPS sont indifférenciés tout comme l'est le CAPEPS. Pourtant, une enquête réalisée par le ministère montre que pour autant ce sont les garçons ou les hommes qui réussissent le mieux à ce concours ainsi qu'à ceux de l'agrégation externe et interne. Bien entendu, la valorisation des pratiques sportives joue certainement un rôle non négligeable. Mais on peut aussi déceler dans cette dissonance par rapport aux autres CAPES et agrégations (où les femmes réussissent mieux que les hommes dans la plupart des sections) un programme caché d'éducation (sportif) qui, ici, touche les enseignants d'EPS spécifiquement. Ce programme caché est pour autant un bon indicateur du masculin - neutre ou du masculin - atténué mis en œuvre de manière dissimulée.

Michel FIZE (2003), vivement critiqué par Michèle ZANCARINI-FOURNEL (2004) pour ses raccourcis historiques et ses conclusions hâtives, montre que les filles réussissent à peu près partout, maintenant, mieux que les garçons dans le système éducatif mixte. Autrement dit, comme dans un retournement inattendu, serait en train de se mettre en place, dans l'école un féminin - neutre ou un féminin - atténué. Cela, pourtant, n'est pas vrai pour toutes les disciplines, puisque l'EPS continue de discriminer les filles. Par ailleurs, ce n'est pas parce que les filles réussissent mieux que les garçons, au plan strictement scolaire, que des programmes cachés, apprenant par exemple aux garçons à être plus combatifs, ne sont pas mis en place. Ce qui expliquerait ensuite la difficulté des filles à «briser le plafond de verre ", c'est-à-dire à accéder à des métiers socialement plus valorisés.

De fait, la féminisation du corps enseignant et importante, mais ce n'est pas une réelle nouveauté, en effet, si aujourd'hui il y a 78,8\% d'enseignantes dans le premier degré ; $56,7 \%$ dans le second degré ; $34,2 \%$ dans le supérieur, dix ans avant, les taux étaient respectivement de $75 \%, 56 \%$ et $28 \%$. Autrement dit, on ne peut pas penser que la possible discrimination des garçons (ils sont 70000 environ de moins que les filles à obtenir le baccalauréat aujourd'hui) puisse être liée à un accroissement significatif du nombre des enseignantes. Par ailleurs, ces chiffres permettent aussi de constater où se situe le "plafond de verre », puisque les universitaires sont bien moins nombreuses que leurs collègues hommes.

En EPS, la discrimination des filles qui vaut pour les enseignants (réussites aux concours), vaut aussi pour les élèves. La mixité est très loin d'être l'égalité. En effet, plusieurs études (COMBAZ, G. 1992, DAVISSE \& LOUVEAU, 1999, VOLONDAT 2000, FIZE, 2003, COGERINO \& TERRET, 2006) successives réalisées sur les notes des élèves au bac montrent que pour une moyenne en général assez élevée (aux environ de 13,5) et en cela peu sélective, les filles ont toujours (cela varie un peu selon les années) entre 0,8 et 2,3 points de moins que les garçons pour une épreuve dotée aujourd'hui (depuis 1995) d'un coefficient non 
négligeable au premier groupe d'épreuves (2) et, surtout sur des très grands nombres d'élèves (près de 400 000).

Beaucoup d'explications ont été avancées à ce sujet, il semble pourtant que la plus simple est rarement évoquée par les analystes de la question : la sportivisation des épreuves. En effet, si l'on observe les pratiques physiques présentes dans les programmes et généralement proposées aux élèves, on doit constater que le sport (à distinguer de l'activité physique, du jeu et de la danse par exemple) y occupe la plus grande place et, qu'en outre, les sports « genrés » masculins sont les plus présents dans les programmes et dans la réalité des mises en œuvre.

Ainsi si l'on prend la liste des activités présentes dans les programmes de classes de 1ère (ensuite présents au bac) et considérées comme les plus couramment enseignées, on peut constater que le plus grand nombre consiste en des activités valorisées par des stéréotypes de genre masculin dans le monde du sport fédéral (il suffit de compter le pourcentage de garçons et de filles inscrits dans les fédérations). Ainsi si le volley-ball ( $\mathrm{F}=$ $48 \%$ ) et le basket-ball $(\mathrm{F}=47 \%)$ peuvent être considérés comme " non genrés » dans la mesure où le nombre des pratiquantes fédérales est assez proche de celui des pratiquants, il n'en va pas de même du hand-ball ( $\mathrm{F}=35 \%)$, du football $(\mathrm{F}=1 \%)$, du rugby $(\mathrm{F}=2 \%)$ et même du badminton $(\mathrm{F}=38 \%)$, du tennis de table $(\mathrm{F}=30 \%)$, de la course d'orientation ( $\mathrm{F}=20 \%$ estimé sur chiffres locaux les chiffres nationaux sont inaccessibles), du judo ( $\mathrm{F}=$ $23 \%)$, de la lutte $(\mathrm{F}=12 \%)$ et de l'athlétisme ( $\mathrm{F}=36,5 \%)$. A l'inverse la natation ( $\mathrm{F}=54 \%)$, sans doute à cause de la natation synchronisée, la gymnastique artistique $(\mathrm{F}=95 \%)$ la gymnastique acrobatique $(\mathrm{F}=79 \%)$ et la danse $(\mathrm{F}=98 \%)$, sont extrêmement féminisées dans le domaine fédéral. Pour deux activités l'escalade ( $\mathrm{F}=$ non disponible) et la course en durée (n'existant pas dans le fédéral), il n'est pas possible de savoir le rapport pratiquantes/pratiquants.

Cependant évidemment, si l'on fait le total des activités massivement investies par les hommes et celles massivement investies par les femmes, dans le domaine fédéral, on peut constater qu'il y a 9 activités physiques masculinisées, 4 activités physiques féminisées, 2 activités physiques non - genrées socialement et deux activités physiques dont les chiffres ne sont pas disponibles (on pourrait toutefois penser que la course de durée pourrait être non genrée). Il est dès lors aisé de comprendre que ces activités sont un programme caché (bien que très visible) d'éducation ou tout au moins de discrimination. On notera d'ailleurs, que l'une des activités physiques les plus féminisées dans la société, le "stretching», n'est pas présente dans la liste, alors que la Fédération Française d'Éducation Physique et de Gymnastique Volontaire, qui licencie plus de 550000 membres, est la troisième fédération sportive française, par le nombre de ses licenciés (après le football et le tennis) et qu'elle propose de la gymnastique d'entretien, très valorisée dans les stéréotypes de genre féminins aujourd'hui.

On voit donc que le programme caché d'éducation peut parfois être très visible (il l'est parfois moins lorsqu'il s'agit de pratiques enseignantes plus discrètes) et qu'il serait très simple d'y remédier, mais que cela n'est pas fait par les instances dirigeantes. Une étude fine des enseignements proposés aux filles et aux garçons dans les autres disciplines (partiellement réalisée), voire dans les procédures d'évaluation ou dans les modalités de sélection, permettrait certainement de mettre en évidence des processus et des procédés similaires, c'est-à-dire, en définitive construisant par le biais de la mixité non pas un métissage enrichissant et égalitaire, mais bien une colonisation du féminin par le masculin (comme c'est le cas généralement dans la langue française). 
Le dernier texte paru en EPS, pour les collèges, le 28 Août 2008, affirme :

«L'éducation physique et sportive (EPS), à tous les niveaux de la scolarité, vise la réussite de tous les élèves [je souligne], et contribue, avec les autres disciplines, à l'instruction, la formation et l'éducation de chacun. Elle participe à l'acquisition et à la maitrise du socle commun et permet de faire partager aux élèves les valeurs de la République. » [...] L'EPS a le devoir d'aider tous les collégiens, filles et garçons [idem] à acquérir de nouveaux repères sur soi, sur les autres, sur l'environnement, de nouveaux pouvoirs moteurs pour construire une image positive de soi. [...] Le projet pédagogique doit présenter une programmation exigeante, équilibrée et suffisamment diversifiée pour permettre aux filles comme aux garçons de réussir [idem] et d'acquérir une culture commune ».

En ce sens il peut être perçu comme soucieux de l'équité voire de l'égalité filles garçons, mais il ne règle pas du tout le problème soulevé ci-dessus. En effet, lorsque l'on étudie la liste des activités physiques proposées, on constate que la grande majorité est encore sportive et surtout valorise les activités sportives genrées masculines dans un rapport 2 / $3,1 / 3$.

1 Pour autant, toutes ces analyses ne doivent pas conduire, comme le fait Fize (2003) à remettre en cause la mixité, bien au contraire. Tout d'abord parce que les enseignantes et enseignants d'EPS soucieux de métissage enrichissant, par le biais de la mixité et non de co-instruction, ont depuis longtemps trouvé des solutions pour permettre à tous les élèves d'avoir une pratique équitable si ce n'est égalitaire. Par exemple en alternant des activés genrées masculines et féminines dans l'horaire légal d'E.P.S. (par exemple $2 \mathrm{~h}$ de danse ou d'expression corporelle et $2 \mathrm{~h}$ de lutte, en classe de 6ème) avec des activités plus neutres (volley-ball, basket-ball, course de durée, jeux traditionnels...), mais aussi en démixant temporairement au cœur de la pratique pour permettre une meilleure appropriation pour tous les élèves, ou encore en modifiant les règles sportives ou en adoptant des stratégies permettant à tous de jouer ensemble (modification, adaptation pour certains en fonction du niveau de pratique et non du sexe, des critères de jeu). Bref, pour beaucoup d'enseignants d'EPS aujourd'hui la mixité est bien un métissage, selon son étymologie, c'est-à-dire un enrichissement symbolique mutuel et non une contrainte.

2 On voit aussi encore ici, que l'EPS est un bon analyseur du reste du système scolaire, même si, parfois, touchant spécifiquement au corps et dérivant vers le sport elle peut être dissonante.

\section{Conclusion}

103 Le but de cet article était de réaliser conjointement un historique de la mixité dans l'éducation en France et plus spécifiquement en éducation physique (et sportive) en glissant, parfois, du côté de l'éducation comparée sans que cela soit une fin en soi ni une contrainte.

104 Il a permis de constater, d'une part, que si la mixité apparaît tardivement dans le système éducatif français officiellement, officieusement elle semble bien plus précoce et répandue que l'on pourrait le croire, y compris au début du XXe siècle. Les classes rurales souvent à plusieurs niveaux, les cours complémentaires, pour simplifier on dira le système primaire, sont le plus souvent mixtes, probablement tout comme le système d'enseignement supérieur à partir du moment où l'on autorise les filles à y accéder. À 
l'inverse, le système secondaire l'est probablement moins du fait de ses origines cléricales, catholiques.

La situation semble assez similaire dans le domaine de la gymnastique puis de l'éducation physique. À l'inverse, le sport fédéral exacerbe les stéréotypes de genre et quand l'éducation physique devient éducation physique et sportive peut-être entre-t-elle en dissonance avec le reste du système éducatif, en promouvant massivement des stéréotypes de genre masculin ou, à tout le moins, un masculin - neutre ou en promouvant, pour les filles, un masculin - atténué (par exemple une course de $30 \mathrm{~m}$ pour les filles de $60 \mathrm{~m}$ pour les garçons, un poids de $3 \mathrm{~kg}$ pour les filles et de $5 \mathrm{~kg}$ pour les garçons, etc.). On rappellera d'ailleurs, sur ce point, que c'est plutôt par le système secondaire, le plus démixé dans l'entre-deux-guerres, que le sport pénètre l'éducation physique (les lycées parisiens de garçons).

D'autre part, ce travail montre comment la mixité n'est pas vecteur d'égalité, aussi bien pour les filles discriminées par le sport en EPS au baccalauréat que pour les garçons discriminés par les notes à ce même diplôme et, plus généralement, dans le système scolaire aujourd'hui.

Pour autant, la question qui a animé les médias un moment, il y a quelques mois concernant la possibilité de remettre en cause la mixité pour des raisons d'égalité semble devoir ne pas être tranchée de manière simpliste, si l'on admet notamment que des solutions peuvent être trouvées à l'inégalité et que les pratiques enseignantes ont déjà, en grande partie, aussi bien en EPS que dans d'autres disciplines, fourni ces solutions. Le simple fait que les futurs enseignants formés dans les IUFM aient des modules consacrés au "genre» dans l'éducation depuis quelques années (à partir de 2001, surtout), contribue à modifier les visions et les pratiques.

De nombreuses recherches sont également mobilisées dans ce domaine. Notons, par ailleurs, que les trois concours de recrutement d'EPS (dans l'ordre agrégation externe, agrégation interne et CAPEPS) ont proposé dans l'épreuve écrite I des sujets sur le genre, le sexe des élèves voire sur la mixité et même le masculin et le féminin (sujet CAPEPS écrit I, 2010). On peut donc penser qu'une prise de conscience est en cours.

Enfin, il faut signaler que les études réalisées sur des classes démixées aux États-Unis d'Amérique, sont loin d'être concluantes pour ce qui est du rétablissement d'une supposée égalité des sexes. En effet, si plusieurs travaux notamment anglo-saxons font le constat d'un renforcement des stéréotypes de genre par la mixité, d'autres (notamment Marie DURU-BELLAT, 2004) montrent que les stéréotypes de sexe n'épargnent pas les écoles non - mixtes. Dans des classes de filles, des enseignantes font parfois des allusions sexistes à l'encontre des femmes ou tendent à simplifier les contenus (surtout dans les matières scientifiques) comme s'il s'agissait de les leur rendre accessibles. Et dans les classes de garçons, on observe parfois des encouragements, de la part d'un enseignant masculin, à une certaine agressivité. Bref, l'égalité n'est pas davantage donnée par le démixage.

Métis sait plus de chose que tout dieu ou mortel, elle est la personnification de la sagesse et de l'intelligence rusée. Si la mixité est bien (ou en voie de devenir), comme on peut le penser, un métissage et non une colonisation d'un genre par l'autre, elle ne peut donc que faire progresser les deux sexes et les enrichir culturellement et symboliquement, il reste à formater des structures et des processus, voire des procédures et des contenus 
d'évaluation (comme on l'a vu pour l'EPS) qui permettent une réelle mise en œuvre et une limitation de certaines dérives inégalitaires qui lui incombent.

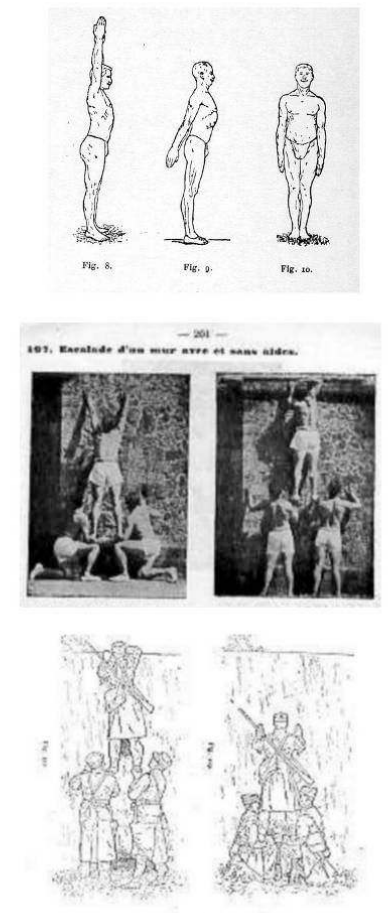

Images de manuels scolaires d'éducation physique. Manuel d'exercices physique et de jeux scolaires, 1907. Règlement général d'Education Physique, Méthode Française, 1925. Projet de règlement général d'Education physique, méthode française, 1921, T2. Libres de droits

\section{BIBLIOGRAPHIE}

AB DER HALDEN, C. (1935), Leçons de morale. Ecoles primaires supérieures, Paris, Armand Colin.

ANDRIEU, G. (1990), L'Éducation physique au XXe siècle une histoire des pratiques, Paris, Actio.

ATTALI, J. \& SAINT-MARTIN, J. (2005), Les oubliés de la démocratisation scolaire de l'éducation physique française (1959-1967), in SAINT-MARTIN, J \& TERRET, T. Sport et genre, T. 3, Paris, L'Harmattan, 207-226.

BAUDELOT, C. \& ESTABLET, R. (1992), Allez les filles! Paris, Seuil.

BERNARD, M. (1974), Le Corps, Paris, Delarge.

BROHM, J.-M. (1975), Corps et politique, Paris, Delarge.

COGERINO, G. (2006), La Mixité en éducation physique. Paris, EPS.

COMBAZ, G. (1992), Sociologie de l'éducation physique, Paris, PUF.

DAVISSE, A. (1999), Elles papotent, ils gigotent - L'indésirable différence des sexes..., Ville, Ecole, Intégration, 116, mars, Paris, 185-198.

DAVISSE , A. \& LOUVEAU, C. (1998), Sports, École, Société : la différence des sexes, Paris, L'Harmattan.

DE COUBERTIN, P. (Baron de, 1905), La Gymnastique utilitaire, Paris, Alcan. 
DE COUBERTIN P. (Baron de, 1912), Discours d'ouverture des jeux olympiques. In : Berlioux, M., D’Olympie à Mexico, Paris, Flammarion.

DEMANIJ, G. (1902), Les Bases scientifiques de l'éducation physique, Paris, Baillières.

DURU-BELLAT, M. (rééd. 2004), L'école des filles : quelle formation, pour quels rôles sociaux? Paris, L'Harmattan.

FILLIOUX, J. (1974), Du contrat pédagogique, Paris, Dunod.

FIZE, M. (2003), Les Pièges de la mixité scolaire, Paris, Presses de la Renaissance.

FONSSAGRIVES, J-B. (1869), L'éducation physique des jeunes filles, Paris, Hachette et Masson.

GAUTHIEZ-RIEUCAU, D. (2005, dir.), Actes du Colloque Quelles mixités construire à l'école ? IUFM de Montpellier, 17 novembre 2004.

GAUTIER, G. (2004), Rapport du Sénat sur la mixité menacée. n²63, 13 avril 2004. Senat, Paris, consultable sur le site du Sénat.

GAY-LESCOT, J.-L. (1999), Éducation physique et sport scolaire entre les deux guerres (1919-1939). Des textes à la réalité, in : L'Éducation physique au XXe siècle (sous la direction de J. GLEYSE), Paris, Vigot, p. 59-68.

GLEYSE J. (1996), L'image de la femme dans les discours sur l'EP et le sport de 1870 à 1930. «Un fait social total »?, in ARNAUD, P. \& TERRET, T., (dir.), Histoire du sport féminin. Sport masculin-sport féminin : éducation et société. T. 2, Paris, L'harmattan.

GLEYSE J. (2008), Os manuais escolares franceses de educaçao fisica, de higiene e de moral seriam sexistas (1880-2004), Educaçao e sociedade, 102, 29, Jan/Abril, CEDES, Campinas, SP, 137-159.

GLEYSE , J. (1997), L'Instrumentalisation du corps. Paris, L'harmattan.

HEBERT, G. (1911), Le Code de la force, Paris, Vuibert.

HEBERT , G. (1919), Muscle et beauté plastique féminine, Paris, Vuibert.

HEBERT , G. (1936), L'Éducation physique virile et morale par la méthode naturelle, Paris, Vuibert.

LABBE, M. (1930), Traité d'éducation physique, Paris, Larousse.

LAPASSADE, G. \& SCHERRER, R. (1974), Le Corps interdit, Paris, ESF.

LE BOULCH, J. (1966), L'Éducation par le mouvement, Paris, ESF.

LE BOULCH, J. (1971), Vers une science du mouvement humain, Paris, ESF.

LE BOULCH, J. (1977), Face au sport, Paris, ESF.

LELIEVRE, F. \& LELIEVRE, C. (1991), Histoire de la scolarisation des filles, Paris, Nathan.

LOUVEAU, C. (2000), Femmes sportives, corps désirables, Le Monde Diplomatique, Paris.

MOSCONI, N. (1989), La Mixité dans l'enseignement secondaire. Un faux-semblant. Paris, PUF.

PARLEBAS, P. (1967), L'Éducation physique en miettes, EPS, 85, Paris, EPS.

PARLEBAS , P. (1985), Lexique commenté en sciences de l'action motrice, Paris, Privat.

PROST, A. (1992), Éducation, société et politique, Paris, Seuil.

PUJADE-RENAUD, C. (1983), Le Corps de l'élève dans la classe, Paris, ESF.

PUJADE-RENAUD , C. (1983), Le Corps de l'enseignant dans la classe, Paris, ESF. 
ROBENE, L. (2005), Itinéraire d'une « prof de gym ». Paulette Morisson (1914-2000), in SAINTMARTIN, J. \& TERRET, T. (2004), Sport et Genre, Volume 3. Apprentissage du genre et institutions éducatives, Paris, L'Harmattan, 319-337.

ROLLIN, F. (1987), La mixité à l'école - Premier bilan, Études, déc 1987, 609-621.

SOHN, A.-M. (2003), Les « relations filles-garçons »: du chaperonnage à la mixité (1870-1970), Travail, Genre et Sociétés, 9, avril, Paris, 91-109.

TERRET, T., COGERINO, G., ROGOWSKI, G. (2006), Pratiques et représentations de la mixité en EPS, Paris, EPS, AFRAPS.

ZAIDMAN, C. (1996), La Mixité à l'école primaire, Paris, L'Harmattan.

ZANCARINI-FOURNEL, M. (2005), Histoire des femmes en France, XIXe-XXe siècle, Rennes, PUR.

\section{NOTES}

1. Il y a un cours complémentaire par niveau, 3 années après le Cours Supérieur et le Certificat d'Etude Primaire ou « Diplôme National » pendant la guerre, et non 4 comme en collège.

\section{RÉSUMÉS}

L'histoire de l'éducation physique par son rapport direct aux corps est un excellent analyseur de l'histoire des Ecoles en France au regard de la question du genre. Elle permet notamment de poser la question de la co-instruction, de la co-éducation et de la mixité comme métissage. Elle favorise également la compréhension d'une tendance historique récemment remise en cause ou à minima, rediscutée aussi bien en France qu'aux Etats-Unis d'Amérique et en Europe. En effet, au cours des toutes dernières années, ce qui semblait une évidence depuis les combats féministes des années 1970 pourrait être remis en cause et l'est parfois. La raison en étant l'accroissement des discriminations visant soit les garçons soit les filles dans les classes mixtes.

History of physical education, in her direct link with body is a good witness of schools history in France, looking to gender question. She allowed, so far, questioning co-instruction, co-education and « mixing of sexes » as breading. She favours equally understanding of an historical tendency recently discussed in France as well as in USA and Europe. In effect, during the very close last years, what seems an evidence since feminists fights of the seventeen's could be questioned and is sometimes questioned. The reason could be increasing of discriminations aiming either boys or girls in mixing sexes classes.

\section{INDEX}

Mots-clés : corps, éducation physique, genre, mixité, stéréotype

Keywords : body, co-education, gender, physical education 


\section{AUTEUR}

\section{JACQUES GLEYSE}

Professeur des Universités en STAPS, IUFM de l'Université de Montpellier 2 